\title{
Cantilever dynamics in amplitude modulation AFM: continuous and dis-continuous transitions
}

Sergio Santos ${ }^{1}$, Victor Barcons ${ }^{2}$, Josep Font ${ }^{2}$, Neil H Thomson ${ }^{1}$.

\author{
${ }^{1}$ School of Physics and Astronomy \& Astbury Centre for Structural Molecular Biology, \\ University of Leeds, LS2 9JT, UK. ${ }^{2}$ Departament d'Enginyeria Electrònica, UPC - Universitat \\ Politècnica de Catalunya Av. Bases, 61, 08242-Manresa, Spain
}

\begin{abstract}
Transitions between the attractive and the repulsive force regimes for amplitude modulation AFM can be either dis-continuous, with a corresponding jump in amplitude and phase, or continuous and smooth. During the transitions, peak repulsive and average forces can be up to an order of magnitude higher when these are discrete. Under certain circumstances, for example when the tip radius is relatively large (e.g. R>20-30nm), and for high cantilever free amplitudes (e.g. A>40-50nm), the L-state can be reached with relatively low set-points only (e.g. $\mathrm{A}_{\mathrm{sp}} / \mathrm{A}_{0}<0.30$ ). We find that these cases do not generally lead to higher resolution despite the fact that the imaging can be non-contact.
\end{abstract}

Running Title: Force Transitions in AM AFM 
The net force between the tip and the sample is a convolution of several forces such as van der Waals, short range repulsive, capillary, adhesion, electrostatic and magnetic among others (depending on the set-up). While these are all fundamentally electromagnetic in nature, they have different distance dependencies that allows the separation of the effects of forces or interactions on the basis of distance ${ }^{1,2}$. Nevertheless, for simplicity, the potential between the tip and the sample can be more simply represented as a non-linear and non-monotonic potential presenting a LennardJones (L-J) shape ${ }^{3}$; this is the potential we have used in the present article. Surprisingly perhaps, a simple point mass model has allowed elucidation of the main characteristics of $\mathrm{AM} \mathrm{AFM}{ }^{4,5}$, interpretation of phase contrast ${ }^{6,7}$ and some understanding of the role of the capillary neck forming between the tip and the sample in ambient conditions ${ }^{8}$. The non-linearity of the potential was first reported to be responsible for the bi-stable behaviour of an STM probe when vibrated over a surface near resonance by Gleyzes et al. ${ }^{9}$. Since then several groups have investigated these effects ${ }^{10-14}$. A major outcome of the modelling work is that either one or two (stable or physically reachable) solutions to the differential equation might exist ${ }^{2,4,15}$. When two solutions exist, these are termed the High (H) and Low (L) state respectively and correspond to two different cantilever amplitude branches for a given equilibrium tip-sample distance $\left(z_{c}\right)$. In addition, a net attractive and a net repulsive average force per cycle define what are commonly termed the attractive and the repulsive force regimes ${ }^{16}$ and, with some exceptions ${ }^{2}$, these correspond to the L and the H-state when they exist. Nevertheless, we emphasise that the attractive and the repulsive force regimes can also be defined when a single branch exists, thus, a transition between force regimes might or might not involve a switch between 
amplitude branches ${ }^{16}$. In fact, there are several fundamental differences between force transitions and state transitions from a physical point of view. In short, a force transition has to be defined whereas the existence of states is a fundamental property of the system. For example, initially, force transitions were defined as purely non-contact to intermittent contact force per cycle whereas the net attractive to net repulsive force transition is now more commonly used. On the other hand, the dynamics of the cantilever dictate whether there is one, two or more physically available oscillation states and this is not subject to definition, that is, it is an inherent characteristic of the system that can be explained mathematically as a multiple solution (double for bi-stability) to the differential equation governing the motion. Additionally, it turns out that for a microcantilever vibrating near a surface and for the typical parameters used in dynamic AFM the transition between states has to be discrete (i.e. step-like changes in amplitude and phase have to occur) and it is stochastic in nature (e.g. the transition might be caused by any noise resulting in an error or perturbation in amplitude). In fact, the latter convention (e.g. force transitions as transitions from net attractive to net repulsive forces per cycle) has become more common, partly because it allows 1) distinguishing between the $\mathrm{L}$ and the $\mathrm{H}$-state when these exist and 2) these can be experimentally monitored by recording the phase (e.g. phase shifts above (below) $90^{\circ}$ correspond to the attractive (repulsive) regime) ${ }^{6,7,15,16}$. However, while phase shifts always follow the net force in this way, large energy dissipation in the tip sample junction might impede distinguishing between the $\mathrm{L}$ and the $\mathrm{H}$-state. This is because even though these might still co-exist when severe dissipation takes place, both might lie either in the repulsive or in the attractive regime. Nevertheless, even in these situations Amplitude and Phase Distance curves (APDs) may still be used 
to differentiate between oscillation states by monitoring the phase shift. Here we have carried out all images by setting the operational parameters while the cantilever is free and then engaging and setting the desired set-point. The amplitude of the free cantilever and the tapping amplitude when the cantilever interacts with the surface are termed free amplitude $\left(\mathrm{A}_{0}\right)$ and amplitude set-point $\left(\mathrm{A}_{\mathrm{sp}}\right)$ respectively.

In this article we demonstrate and discuss differences between smooth and step-like transitions from the attractive to the repulsive regime, and show that the forces involved are of different magnitudes. We consider the two most common scenarios in AM AFM; 1) regions where a single branch exists (Figure 1) and 2) regions where bi-stability is present and the amplitude set-point (and/or free amplitude and/or drive frequency) has to be adjusted to avoid and/or control bistability (Figure 2). We also report that L-state imaging with relatively small amplitude set-point ratios $\left(\mathrm{A}_{\mathrm{sp}} / \mathrm{A}_{0}<0.3\right)$, when it is experimentally unreachable at intermediate and/or high values of set-point, does not generally lead to improved resolution. We use dsDNA on mica in ambient conditions as a model system in AM AFM. A smooth transition between the attractive and the repulsive force regime is shown in Fig. 1. As stated, it is well known that the average force per cycle is associated with phase shifts $(\Phi)$ above and below $90^{\circ}$ for the attractive and the repulsive force regime respectively. This is particularly true when the set-point is not too small (e.g. $A_{\text {sp }} / A_{0}>0.3$, see Ref. 2 and our Figure 2). In addition, a switch between states involves a small perturbation in A and a step-like shift in $\Phi$ and $z_{c}$ (see Fig. 2). However, cases exist where neither the topographic (Fig. 1a) nor the phase contrast (Fig. 1b) images show a discrete step as the phase shifts from $\Phi>90^{\circ}$ (bottom of the scan) to $\Phi<90^{\circ}$ (top 
of the scan). Amplitude (Fig. 1d) and phase (Fig. 1e) distance (APD) curves readily allow experimental verification that in this case the transition is smooth. Note that a smooth transition in phase, occurs both on z-piezo extension and retraction, with no signs of hysteresis. A region with negative slope can be observed in the amplitude curve (Fig. 1d) in the region close to where the phase crosses $90^{\circ}$ indicative of a single branched region as previously reported for APDs ${ }^{2,16,17}$. This negative slope is smooth and deterministic and does not imply the onset of intermittent contact from purely non-contact nor it is due to adhesion as can be shown with the use of the spring model and an L-J shaped potential. Indeed, the model shows that this negative slope always coincides with a smooth increase in the repulsive net force (and decrease in phase shift) with decreasing $\mathrm{z}_{\mathrm{c}}$ (data not shown). Nevertheless intermittent contact already occurs before (e.g. for higher values of $z_{c}$ ) this negative slope starts. Furthermore, it has been reported that this negative slope region might cause instabilities and/or artefacts when using an AFM with an amplitude feedback ${ }^{2,17}$. Nevertheless, in the single branch region the cantilever can, in principal, be driven from the repulsive to attractive force regime (irrespective of sign of the slope in amplitude), for example with z-piezo modulation. Hence, the behaviour here is drastically different from the situation when two oscillation branches exist, where no form of feedback could control a switch between states. Note that when we speak about controlling bi-stability we are in fact choosing operational parameters for which one or the other state is highly stable rather than controlling the switch itself (i.e. we seek to avoid the bi-stable region). A line-section (Fig. 1c) of the phase image across the transition region (dashed line in Fig. 1b) shows that the transition takes place for a distance of approximately $250 \mathrm{~nm}(\sim 40 \mathrm{sec})$ in the slow scan direction. When bi-stability 
is not present, a shift in phase for a constant set of operational parameters and cantilever properties is always associated with a change in energy dissipated in the tip-sample interaction $^{7}$ and indicates a change in either the local environment or the properties of the sample. ${ }^{6-8,15}$ At intermediate values of relative humidity, the surface of mica is extremely active and the height of the water layer and the concentration of salts and other impurities might rapidly vary ${ }^{18,19}$. Since the image was taken at $60 \%$ relative humidity it is reasonable to think that such activity has been the trigger of the smooth phase shift.

Next, we show an experimental example of a transition from the $\mathrm{H}$ to the L-state for small set-point ratios (e.g. $A_{\mathrm{sp}} / A_{0}<0.3$ ). In this case, a step-like discontinuity is observed both in the topographic (Fig. 2a) and the phase contrast (Fig. 2b) images. The discontinuity can also be observed in the corresponding APD curves (Fig. 2e and f). This is a characteristic transition between states, except for the fact that both the $\mathrm{L}$ and the $\mathrm{H}$ state occur when the phase is greater than $90^{\circ}$ due to severe inelastic interactions ${ }^{6,7,16}$. That is, this is a transition between states but not a transition between force regimes. We can establish that a switch between states has occurred by comparing the average phase shift in the scans (Figure 2b) with the phase shift in the APD curves (Figure 2f); markers ( $\mathrm{H}$ and L) are placed in the phase curve (Figure 2f) for the set-point used in the scan (Figures 2a-b). There is considerable loss of contrast in the L-state, both in topography and phase. We have consistently had the same outcome for over 50 probes and the situation for which the L-state can only be physically reached with small set-points $\left(\mathrm{A}_{\mathrm{sp}} / \mathrm{A}_{0}<0.3\right)$ occurred only when using relatively high free amplitudes $\left(\mathrm{A}_{0}>40-50 \mathrm{~nm}\right)$. This situation generally coincides with higher instabilities and loss of contrast in the L- 
state compared to the H-state (see Fig. 2) and the requirement of high critical (minimum) free amplitudes $\left(\mathrm{A}_{0}>30-35 \mathrm{~nm}\right)$ to reach the $\mathrm{H}$-state at resonance even with stiff cantilevers $(k>20 N / m)$; note that the critical free amplitude $\left(A_{c}\right)$ to reach the H-state is defined here as the minimum $\mathrm{A}_{0}$ required to observe switching to the H-state in APD curves at resonance. The above indicates that a large tip radius $(\mathrm{R})$ might be required to experimentally observe such behaviour, since $\mathrm{R}$ controls $\mathrm{A}_{\mathrm{c}}$ for a given cantilever-sample set-up. $^{8,15}$. We have confirmed this by imaging the AFM tips with an SEM (data not shown) and, in all cases (provided $\mathrm{k}>20 \mathrm{~N} / \mathrm{m}$ ), this behaviour (Fig. 2) could be observed only for R $>20-30 \mathrm{~nm}$. The noise present is not a direct consequence of more energy being dissipated in one or the other state since, as in this example, these situations typically involve more energy being dissipated in the L than in the H-state according to analytical derivations readily available in the literature ${ }^{7}$. For the situation in Figure 2, the calculations show that more energy is dissipated per cycle in the H-state $\left(\Delta \mathrm{E}_{\mathrm{ts}} \sim 500 \mathrm{eV}\right)$ than in the L-state $\left(\Delta \mathrm{E}_{\mathrm{ts}} \sim 50 \mathrm{eV}\right)$. This is true even when allowing for large errors in the parameters involved in the calculation (e.g. $\pm 20 \%$ in $\mathrm{Q}, \pm 20 \mathrm{~N} / \mathrm{m}$ in $\mathrm{k}$ and $\pm 30^{\circ}$ in $\varphi$ ). The difference in energy dissipation between states could also be deduced by looking at the dramatic difference in phase contrast in the $\mathrm{L}$ and $\mathrm{H}$ state (note the scales; $\Delta 1^{\circ}$ in the $\mathrm{L}$ state and $\Delta 30^{\circ}$ in the $\mathrm{H}$-state in Fig. $2 \mathrm{~b}$ ). Hence arguments similar to those used to derive the stability criteria $^{20}$ (Eqn. 1) cannot be used to interpret this common and highly reproducible phenomenon. It is also unlikely that more energy is dissipated into higher harmonics in the L-state than in the H-state since, typically, harmonics are more readily excited in the repulsive regime and/or for larger sample indentations ${ }^{21-23}$. It could also be argued that as the effective resonant frequency shifts to higher frequencies with 
increasing free amplitude ${ }^{4}$ and, usually, decreasing set-point, noise should be observed for these choice of operational parameters (e.g. relatively high free amplitudes and low set-points). Nevertheless, the effective resonant frequency has a single value for a set of operational parameters (except set-point), separation $\left(\mathrm{z}_{\mathrm{c}}\right)$ and cantilever-sample properties ${ }^{4}$, hence the same (or less) noise should be observed in the L-state in these cases according to this argument. This follows from the fact that for a given set-point (as in Figure 2 or any scan in AM AFM) the cantilever vibrates higher above the sample (larger $z_{c}$ ) in the L-state (implying lower resonant frequencies there) than in the H-state, and this (larger $z_{c}$ ) has been theoretically shown to lead to lower effective resonant frequencies (see Ref. 4).

$$
\frac{k}{2}(A s p)^{2} \geq \Delta E_{t s} \frac{Q}{2 \pi}
$$

We propose that the adhesive force, the related cantilever mean deflection and cantilever restoring force and the instabilities involved with switching into the $\mathrm{H}$ state are responsible for the observed instabilities in the L-state in these situations (and the above mentioned), since at relatively long distances (e.g. $>0.5 \mathrm{~nm}$ above the surface) "a snap into contact" can readily occur ${ }^{24}$. The relationships between all these sources of noise are discussed in the remainder of the article. The snap-to-contact, and adhesion on retraction, can be observed in the curves (Fig. 2e-f). However, the noise is not only present in the regions where the snap into contact will occur but also in regions of larger separations or higher set-points and these might be induced by large cantilever deflections there (see the 
deflection for $0.05<A_{\mathrm{sp}} / A_{0}<0.25$ in Figure 3). Since the snap to contact might occur at any point for this range of set-points it might cause instabilities in several ways, for example, by momentarily adhering the tip onto the sample and/or by providing the activation energy, or perturbation in amplitude, necessary to switch into the H-state. However, both end results (e.g. the cantilever adhering to the sample and the cantilever switching states) are two very different scenarios from a physical point of view. Briefly, the snap into contact is a consequence of the cantilever drifting closer to the sample due to either a bad choice of gains or feedback errors, or a perturbation in amplitude that momentarily gets the tip close enough to the surface until it adheres to it (e.g. zero or close to zero set-point). On the other hand, the switch into the H-state requires a perturbing increase in amplitude large enough to allow a full switch between states (e.g. larger, and momentary if rapidly switching back to the L-state, set-point when the cantilever switches to the H-state). However, as stated, perturbations due to one or the other might provide enough energy (or a perturbation large enough) to produce one or the other outcome. These mechanisms can account for instability in these circumstances (Figure 2) and whenever a relatively large tip radius (e.g. R>20-30nm), a compliant cantilever (e.g. $\mathrm{k}<2-10 \mathrm{~N} / \mathrm{m}$ ), large surface energies (or a combination of these) and intermediate to large free amplitudes (e.g. $\mathrm{A}_{0}>10-20 \mathrm{~nm}$ ) are used in AM AFM. Furthermore, since we only observed noise effects such as those represented in Figure 2 when using relatively large tips ( $\mathrm{R}>20-30 \mathrm{~nm})$, this hypothesis is supported by the fact that the force of adhesion is proportional to the effective tip radius ${ }^{25}$. The implications of adhesion (and the attractive force component in general) on cantilever mean deflection are discussed later. It should also be noted that even though a suitable choice of gains is 
important in dynamic $\mathrm{AFM}^{26}$, we find that no choice of gains makes the noise disappear, particularly in the L-state (data not shown). The area shown in Figs. 2a-b was also imaged in the L-state with relatively small free amplitudes $\left(\mathrm{A}_{0}=15 \mathrm{~nm}\right)$ before (Fig. $2 \mathrm{c}$ ) and after (Fig. 2d) performing these high free amplitude scans. The dashed line in Fig. 2d divides the scan between the area imaged in the L-state in Fig. 2a-b (bottom) and the Hstate (top). No significant loss of contrast is observed between top and bottom in Fig. 2d or between the scan taken before and after high free amplitude imaging (cf. Fig. 2c-d). We note that these results are general in our experiments, that is, when $\mathrm{R}>20-30 \mathrm{~nm}$ and, consequently, the dynamics similar to those observed in Fig. 2e-f, no tip or sample damaged could be observed. We interpret these results as the tip having achieved mechanical and chemical stability. Finally, Figures $2 \mathrm{c}-\mathrm{d}$ are also demonstration of how by simply reducing the free amplitude to low or intermediate values (typically $2<\mathrm{A}_{0}<15 \mathrm{~nm}$ according to our experiments), L-state imaging with relatively low noise levels can occur even when using large tips (e.g. R>20-30nm). We have also performed similar experiments below and above resonance for the same free amplitudes as those in Figure 2 (e.g. $A_{0}=15$ and $56 \mathrm{~nm}$ ) and intermediate values $\left(A_{0}=25 \mathrm{~nm}\right)$ for a whole range of set-points (data not shown), and conclude that there are some trends for these mechanically stable tips. First, it should be noted that the noise can be reduced in the Lstate for relatively small free amplitudes by keeping the set-point relatively high while driving close enough to resonance, e.g. keeping the drive frequency around, $\pm 0.1 \%$, that of the natural frequency. Increasing the free amplitude to intermediate or relatively high values (e.g. $10<\mathrm{A}_{0}<50 \mathrm{~nm}$ ) generally allows imaging in the $\mathrm{L}$ state with low noise only above resonance and for intermediate to high set-points $\left(0.3<A_{\mathrm{sp}} / \mathrm{A}_{0}<0.9\right)$. Then for 
relatively high values of free amplitude $\left(\mathrm{A}_{0}>30-50 \mathrm{~nm}\right)$ repulsive imaging is generally observed to reduce noise at and/or below resonance, whether that be via single branched regions as in Figure 1 or via the $\mathrm{H}$ state with intermediate or relatively high set-points with these tips (e.g. R>20-30nm). Finally, in terms of set-point, free amplitude and noise, note that to a first approximation the stored energy of the cantilever is proportional to the tapping amplitude. This approximation is typically used in AFM analysis to investigate cantilever stability and/or consider energy relationships ${ }^{20}$. Then, from this approximation and assuming that the strength of the attractive component increases with decreasing tipsample distances for small to large distances (e.g. $\mathrm{d}>0.1-0.3 \mathrm{~nm})^{1,25,27}$ it is reasonable to state that, as the set-point and/or energy entering the cantilever per cycle decreases (e.g. decreasing $A_{s p}$ and/or $A_{0}$ ), the noise should increase. However, while the first statement typically agrees with our experiments in the L-state (e.g. decreasing set-points result in increasing noise levels in the L-state, data not shown), the latter (in general and provided $A_{0}$ is not extremely small, e.g. $\left.A_{0}<1-3 \mathrm{~nm}\right)$ does not. Nevertheless, this apparent contradiction can be understood once one realises that, in the L-state, the cantileversample separation $\left(\mathrm{z}_{\mathrm{c}}\right)$ decreases, according to simulations (data not shown), with increasing $\mathrm{A}_{0}$. The implication is that, for a given drive frequency, as $\mathrm{A}_{0}$ increases, the tip is, on average, closer to the sample for a given set-point. This decrease in separation results in the adhesive (or attractive) component of the force gaining strength against the restoring force (or $\mathrm{A}_{\mathrm{sp}}$ from energy considerations) of the cantilever and can generally be deduced, both experimentally and theoretically (see discussion below and Figures 2 and 3) by looking at the mean cantilever deflection for a given cantilever-sample separation and the resulting noise levels. Note that the tapping amplitudes are similar in Figs. 2a-b 
and 2c-d and only the free amplitude (e.g. $A_{0}$ or energy entering the cantilever per cycle) differs from one to the other; the decrease in free amplitude from Figures 2a-b to Figures 2c-d implies that the tip is, on average, closer to the sample (e.g. the cantilever has more negative deflection) in Figures 2a-b than in Figures 2c-d (see discussion below).

We have simulated the tip-sample interaction with the use of a point-mass spring model as detailed in Ref. 16, commercial software ${ }^{28}$ and the classical Runge-Kutta method. The validity of this model in ambient conditions with stiff cantilevers is discussed in the literature ${ }^{23}$. In experimental APD curves the cantilever sample separation $z_{c}$ is varied continuously while both phase and amplitude are monitored. Thus, while some ${ }^{8}$ have solved the equation by first setting a given $z_{c}$ distance and initial conditions and waiting for the steady state response to record phase, amplitude and force, here, the simulations of APD curves have been performed in an analogous way to experiment. We have used $\dot{\mathrm{z}}_{\mathrm{c}}$ speeds ranging from 0.01 to $2 \mathrm{~nm} / \mathrm{ms}$ consistent with typical experiments. We find that the value of $A_{c}$ strongly depends on both $\dot{z}_{c}$ and $z_{c}$ in a very non-linear fashion. This behaviour strongly resembles the real behaviour of the cantilever in experimental APD curves where the switch is intrinsically stochastic and sensitive to any small changes in both operational parameters and set-up.

Fig. 3 shows the outcomes of simulation for a particular set of cantilever-sample properties where the curves resemble very well the experimental behaviour in Fig. 2e-f. Fig. 3 shows the mean tip-sample forces occurring and the amplitude (Fig. 3c) and phase (Fig. 3d) at high set-points. For very small separations $\left(\mathrm{z}_{\mathrm{c}} / \mathrm{A}_{0}<0.03\right)$ the tip adheres onto 
the surface where the mean deflection is approximately $1 \mathrm{~nm}$. For higher separations $\left(0.03<\mathrm{z}_{\mathrm{d}} / \mathrm{A}_{0}<0.23\right)$, the L-state dominates on retraction. Significantly, there is still a considerable amount of negative deflection (approximately $1 \AA$ ), while the average force remains negative and relatively high $(2-3 \mathrm{nN})$. However, for the same range of separations the deflection is smaller in the H-state (Fig. 3a, extension), i.e. the $\mathrm{H}$-state exists with the cantilever closer on average to the equilibrium position. We believe that this larger mean deflection in the L-state is a consequence of adhesion and tip-sample proximity and a source of noise in situations such as that described in Fig. 2, where, while the restoring force on the cantilever is pulling it back towards the H-state with mean forces of the order of nanoNewtons, the adhesive force gains strength against the restoring force in the L-state for a given set-point as the free amplitude increases. Note however, that, as stated, the tip-sample proximity cannot be the only source of noise since the cantilever is always closer, on average, to the surface in the $\mathrm{H}$-state. Hence, as argued above, the observed noise originates from a complex convolution of effects where cantilever deflection plays an important role. This interpretation agrees with experimental results and simulation since, 1) we have observed in the simulations that the mean deflection (mean cantileversample separation) in the L-state decreases (increases) with decreasing free amplitude and 2) experimental imaging in the L-state with no background noise was possible for intermediate to low separations (e.g. $5<\mathrm{z}_{\mathrm{c}}<15 \mathrm{~nm}$ ) provided the free amplitude was kept small enough $\left(\mathrm{A}_{0}<20 \mathrm{~nm}\right)$ (cf. Fig. 2a-b with $\left.2 \mathrm{c}-\mathrm{d}\right)$. While these parameters might change from set-up to set-up, the fundamental relation between increasing free amplitude and increasing deflection in the $\mathrm{L}$ state holds in general, even if a different range of free amplitudes has to be used in other scenarios. For example, for situations of larger surface 
energy the deflections are larger for a given tip radius and free amplitude. Nevertheless, for our set-up, and according to SEM measurements, the range of free amplitudes and tip radii for which the noise was consistent from experiment to experiment was that detailed in the article. Finally, for the parameters in this last simulations, a single branch with respective smooth transitions on both extension and retraction (amplitude, force and phase) and a region with negative slope in amplitude could only be observed experimentally for $A_{0}>150 \mathrm{~nm}$, which is unrealistic for $\mathrm{k}>40 \mathrm{~N} / \mathrm{m}$. In the single branch case for $A_{0}=190 \mathrm{~nm}$, the peak repulsive (average) forces are $30 \mathrm{nN}$ or less (zero) with a dependence on $\dot{z}_{\mathrm{c}}$. When $\mathrm{A}_{0}<150 \mathrm{~nm}$ and bi-stability exists then these forces can be as high as $400-600 \mathrm{nN}(40-50 \mathrm{nN})$ and are independent of $\dot{z}_{\mathrm{c}}$ (Fig. 3).

Finally, we obtained simulated single branched regions at resonance with free amplitudes as low as $25 \mathrm{~nm}$ by reducing $\gamma, \mathrm{H}$ and $\mathrm{R}$ to $30 \mathrm{~mJ} / \mathrm{m}^{2}, 6 \times 10^{-20} \mathrm{~J}$ and $10 \mathrm{~nm}$ respectively. These indicate that for smooth transitions (with intermediate free amplitudes, e.g. $\mathrm{A}_{0}=25 \mathrm{~nm}$ ) such as the one shown in Fig. 1, peak repulsive (average) forces are no higher than $5 \mathrm{nN}$ (zero), whereas for $\mathrm{A}_{0}=8 \mathrm{~nm}$ these can be as high as $30 \mathrm{nN}(2.5 \mathrm{nN})$. These large differences in applied forces imply that step-like discontinuities should be avoided, even when slowly approaching the surface, to prevent tip and sample damage when transitioning into the repulsive force regime. According to our results, this is particularly true when using sharp tips even if using small free amplitudes; this could be due to the high pressures involved when using sharp tips. That is, a large tip radius seems to be stable even if using large free amplitudes (see Fig. 2) to achieve L to $\mathrm{H}$ transitions even though peak forces can reach several hundred $\mathrm{nN}$ whereas sharper tips (e.g. $\mathrm{R}<20 \mathrm{~nm}$ ) 
could potentially degrade under these circumstances even when small free amplitudes, and peak forces, are used. These results should provide insight into carefully controlling peak and average forces to preserve both AFM tips and soft samples, such as biomolecules bound to stiff surfaces.

We would like to thank Bill Bonass and Daniel Billingsley for kindly providing us with dsDNA. Sergio Santos is funded through a Doctoral Training Grant of the BBSRC and Asylum Research Corporation.

J. Israelachvili, Intermolecular \& Surface Forces (Academic Press, 1991).

R. Garcia and R. Perez, Surface Science Reports 47197 (2002).

H. Hölscher, U. D. Schwarz, and R. Wiesendanger, Applied Surface Science 140, 344 (1999).

A. S. Paulo and R. Garcia, Physical Review B 66, 4061 (2002).

R. Stark, G. Schitter, and A. Stemmer, Physical Review B 68, 4011 (2003).

J. Tamayo and R. Garcia, Applied Physics Letters 73, 2926 (1998).

J. P. Cleveland, B. Anczykowski, A. E. Schmid, and V. B. Elings, Applied Physics Letters 72, 2613 (1998).

8 L. Zitzler, S. Herminghaus, and F. Mugele, Physical Review B 66, 155436 (2002).

$9 \quad$ P. Gleyzes, P. K. Kuo, and A. C. Boccara, Applied Physics Letters 58, 2989 (1991).

10 B. Anczykowski, D. Krüger, and H. Fuchs, Physical Review B 53, 15485 (1996). 
J. P. Aimé, R. Boisgard, L. Nony, and G. Couturier, Physical Review Letters 82, 3388 (1998).

M. Marth, D. Maier, and J. Honerkamp, Journal of Applied Physics 85, 7030 (1999).

L. Nony, R. Boisgard, and J. P. Aime, Journal of Chemical Physics 111, 1615 (1999).

L. Wang, Surface Science 429, 178 (1999).

X. Chen, M. C. Davies, R. C.J., S. J. B. Tendler, P. M. Williams, and N. A. Burnham, Surface Science 460, 292 (2000).

R. Garcia and A. San Paulo, Physical Review B 60, 4961 (1999).

R. Garcia and A. San Paulo, Ultramicroscopy 82, 79 (2000).

H. K. Christenson, Journal of Physical Chemistry 97, 12034 (1993).

F. Ostendorf, C. Schmitz, S. Hirth, A. Kühnle, J. J. Kolodziej, and M. Reichling, Langmuir 25, 10764 (2009).

F. J. Giessibl, Reviews of Modern Physics 75, 949 (2003).

R. Proksch, Applied Physics Letters 89, 113121 (2006).

R. W. Stark, Nanotechnology 15, 347 (2004).

T. R. Rodríguez and R. García, Applied Physics Letters 80, 1646 (2002).

Y. Gan, Surface Science Reports 64, 99 (2009).

B. V. Derjaguin, V. Muller, and Y. Toporov, Journal of Colloid and Interface Science 53, 314 (1975).

G. Couturier, R. Boisgard, L. Nony, and J. P. Aimé, Review of Scientific Instruments 74, 2726 (2003).

H. C. Hamaker, Physica 4, 1058 (1937).

T. M. MATLAB R2008a and SIMULINK, Inc., Natick, Massachusetts, US.

T. E. Balmer, H. K. Christenson, N. D. Spencer, and M. Heuberger, Langmuir 24, $1566(2008)$. 


\section{Figure Captions}

FIG 1. An experimental smooth transition. a) Topography and b) phase contrast of dsDNA molecules on mica where a smooth transition from the attractive to the repulsive regime is observed. c) Line-section of the phase image as indicated by the dashed line in b. Corresponding d) amplitude and e) phase distance curves. Insets in (d) show the smooth transition observed experimentally (E) and by simulation (S). The continuous (dashed) line stands for extension (retraction). Slow scan axis upwards. (Experimental Parameters: $\mathrm{A}_{0}=17 \mathrm{~nm}, \mathrm{~A}_{\mathrm{sp}} / \mathrm{A}_{0}=0.75, \mathrm{k}=40 \mathrm{~N} / \mathrm{m}, \mathrm{Q}=550, \mathrm{f}_{0}=318 \mathrm{kHz}$ and $\mathrm{f}=\mathrm{f}_{0}$ ).

FIG. 2. An experimental discontinuous transition at high $\mathrm{A}$ and low set-point. a) Topography and b) phase contrast where a switch from the $\mathrm{H}$ to the L-state occurs for high $\mathrm{A}_{0}$. Topographic scans in the L-state with small free amplitudes $\left(\mathrm{A}_{0}\right)$ taken both $\left.\mathrm{c}\right)$ before and d) after the scans shown in a-b). The dashed line separates the regions scanned in the $\mathrm{L}$ and $\mathrm{H}$-state in the top images respectively. Corresponding e) amplitude and $\mathrm{f}$ ) phase distance curves are shown in extension (continuous line) and retraction (dashed line) with a free amplitude of $\mathrm{A}_{0}=56 \mathrm{~nm}$ at resonance. Insets in (e) show snap-to-contact and adhesion hysteresis for experiment (E) and simulation (S). The "zero" for z-piezo is chosen arbitrarily in the APD curves. (Experimental Parameters: a-b) $A_{0}=56 n m, A_{\mathrm{sp}} /$ $\mathrm{A}_{0}=0.18, \mathrm{k}=40 \mathrm{~N} / \mathrm{m}, \mathrm{Q}=650$ and $\mathrm{f}_{0}=332 \mathrm{kHz}$ and $\left.\mathrm{f}=332 \mathrm{kHz} ; \mathrm{c}-\mathrm{d}\right) \mathrm{A}_{0}=15 \mathrm{~nm}, \mathrm{~A}_{\mathrm{sp}} / \mathrm{A}_{0}=0.80$ $\mathrm{k}=40 \mathrm{~N} / \mathrm{m}, \mathrm{Q}=650$ and $\mathrm{f}_{0}=332 \mathrm{kHz}$ and $\mathrm{f}=332 \mathrm{kHz}$ ). All the images shown here are taken in the attractive force regime whether at low set-point with large amplitude ( $a$ and $b$ ) or lower amplitude and higher set-point (c and d). 
FIG 3. Simulated average tip-sample forces during approach-retract curve, where the cantilever first switches discontinuously to the $\mathrm{H}$-state on extension (continuous black line) and gets trapped onto the surface. On retraction (dashed blue line) it starts trapped in the L-state, then switches to the H-state discontinuously resulting in high transient forces and it finally smoothly returns to the L-state once the H-state ends. Zoomed views of a) mean deflection and b) average force at small separations and c) normalised amplitude and d) phase shift at large separations. Parameters: $A_{0}=63 \mathrm{~nm}, \mathrm{k}=40 \mathrm{~N} / \mathrm{m}, \mathrm{f}_{0}=300 \mathrm{kHz}$, $\mathrm{f}=300 \mathrm{kHz}, \mathrm{E}_{\mathrm{t}}=120 \mathrm{GPa}$ (elastic modulus of the tip), $v=0.3$ (Poisson's coefficient), $\mathrm{H}$ $=2 \times 10^{-19} \mathrm{~J}$ (Hamaker), $\gamma=100 \mathrm{~mJ} / \mathrm{m}^{2}$ (surface energy), $\mathrm{R}=30 \mathrm{~nm}, \mathrm{Q}=500$ and $\mathrm{z}_{\mathrm{c}}=73 \mathrm{~nm}$. Note that these are predicted values for $\mathrm{H}$ and $\gamma$ for mica at $40 \%$ relative humidity ${ }^{29}$. Note that here $\mathrm{H}$ is an energy and should not be confused with the $\mathrm{H}$ state. 

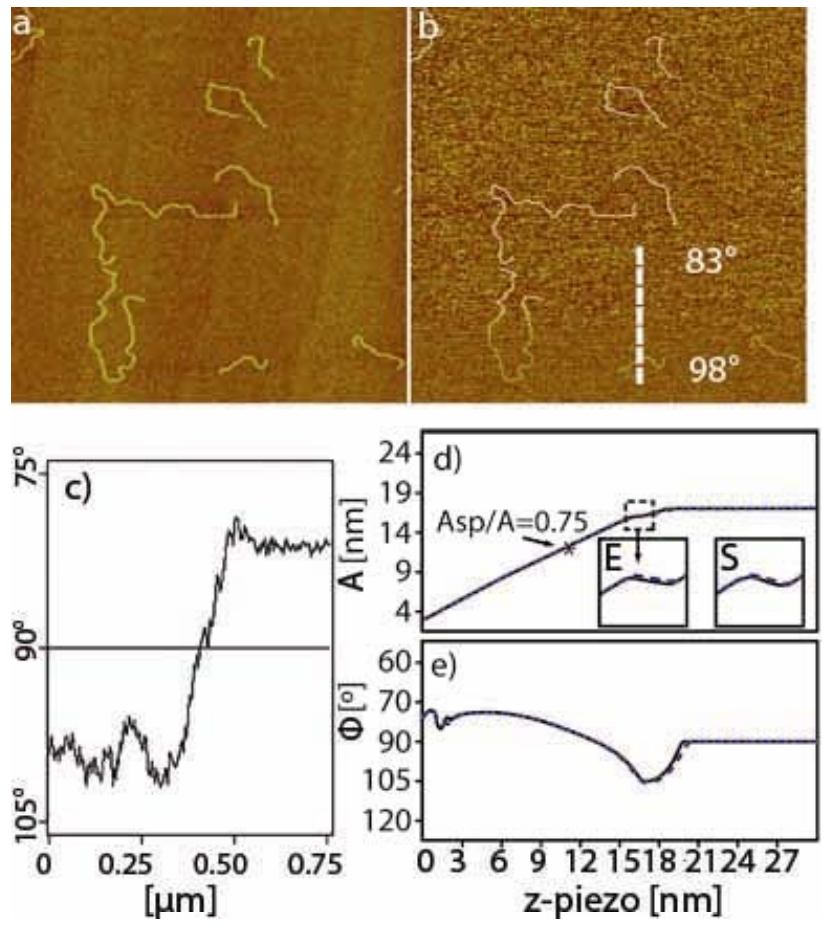


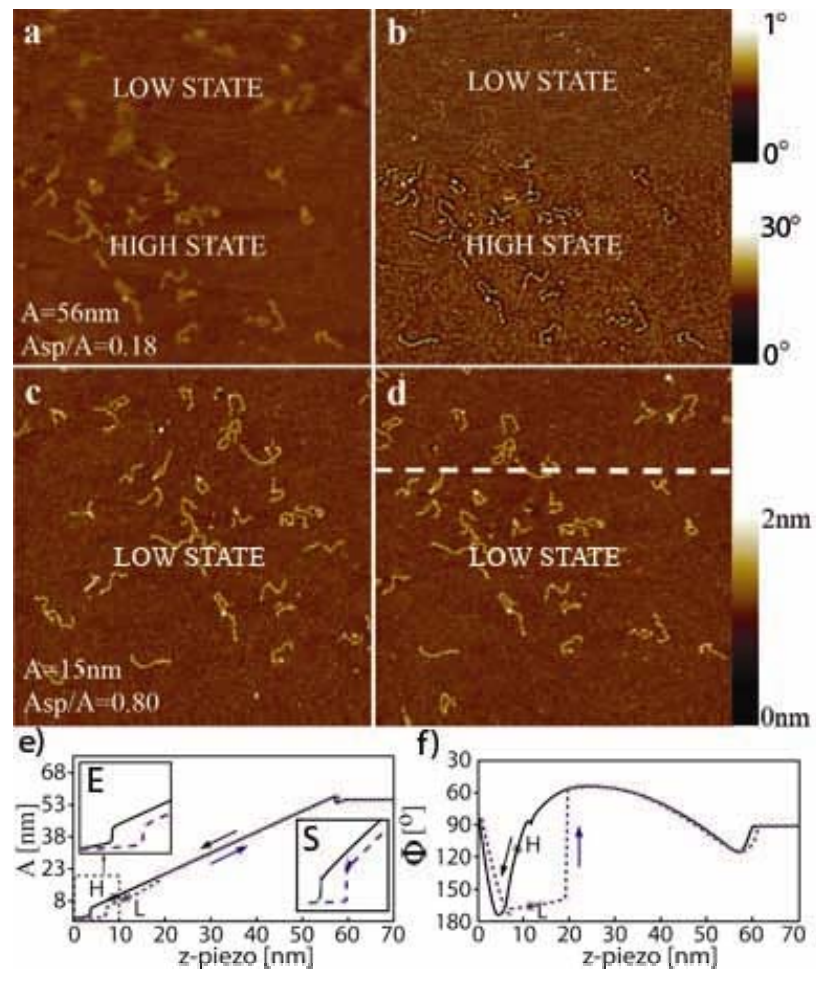




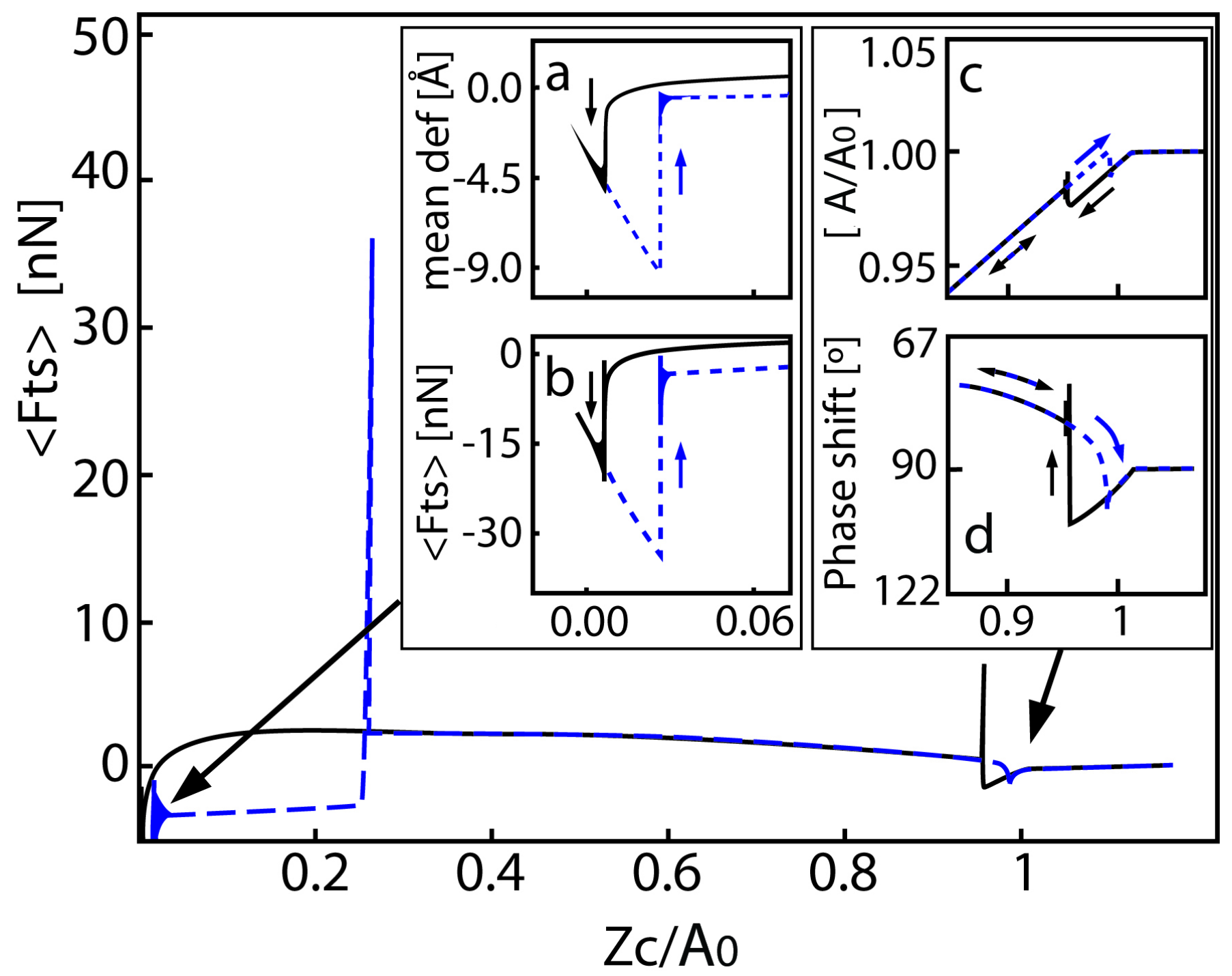

